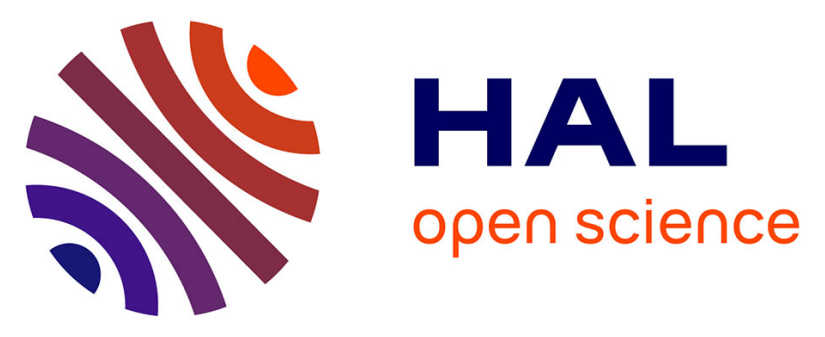

\title{
Speed for the elite, consistency for the masses: differentiating eventual consistency in large-scale distributed systems
}

\author{
Davide Frey, Achour Mostefaoui, Matthieu Perrin, Pierre-Louis Roman, \\ François Taïani
}

\section{To cite this version:}

Davide Frey, Achour Mostefaoui, Matthieu Perrin, Pierre-Louis Roman, François Taïani. Speed for the elite, consistency for the masses: differentiating eventual consistency in large-scale distributed systems. Proceedings of the 2016 IEEE 35th Symposium on Reliable Distributed Systems (SRDS 2016), Sep 2016, Budapest, Hungary. pp.197-206, 10.1109/SRDS.2016.032 . hal-01344138

\section{HAL Id: hal-01344138 \\ https://inria.hal.science/hal-01344138}

Submitted on 11 Jul 2016

HAL is a multi-disciplinary open access archive for the deposit and dissemination of scientific research documents, whether they are published or not. The documents may come from teaching and research institutions in France or abroad, or from public or private research centers.
L'archive ouverte pluridisciplinaire HAL, est destinée au dépôt et à la diffusion de documents scientifiques de niveau recherche, publiés ou non, émanant des établissements d'enseignement et de recherche français ou étrangers, des laboratoires publics ou privés. 


\title{
Speed for the elite, consistency for the masses: differentiating eventual consistency in large-scale distributed systems
}

\author{
Davide Frey*$^{*}$, Achour Mostefaoui ${ }^{\dagger}$, Matthieu Perrin ${ }^{\dagger}$, Pierre-Louis Roman ${ }^{\ddagger}$, François Taïani ${ }^{\ddagger}$ \\ *Inria Rennes, France \\ davide.frey@inria.fr \\ †University of Nantes - LINA, France \\ achour.mostefaoui@univ-nantes.fr, matthieu.perrin@univ-nantes.fr \\ ${ }^{\ddagger}$ University of Rennes 1 - IRISA - ESIR, France \\ pierre-louis.roman@irisa.fr, francois.taiani@irisa.fr
}

\begin{abstract}
Eventual consistency is a consistency model that emphasizes liveness over safety; it is often used for its ability to scale as distributed systems grow larger. Eventual consistency tends to be uniformly applied to an entire system, but we argue that there is a growing demand for differentiated eventual consistency requirements.

We address this demand with UPS, a novel consistency mechanism that offers differentiated eventual consistency and delivery speed by working in pair with a two-phase epidemic broadcast protocol. We propose a closed-form analysis of our approach's delivery speed, and we evaluate our complete mechanism experimentally on a simulated network of one million nodes. To measure the consistency trade-off, we formally define a novel and scalable consistency metric that operates at runtime. In our simulations, UPS divides by more than 4 the inconsistencies experienced by a majority of the nodes, while reducing the average latency incurred by a small fraction of the nodes from 6 rounds down to 3 rounds.
\end{abstract}

\section{INTRODUCTION}

Modern distributed computer systems have reached sizes and extensions not envisaged even a decade ago: modern datacenters routinely comprise tens of thousands of machines [1], and on-line applications are typically distributed over several of these datacenters into complex geo-distributed infrastructures [2], [3]. Such enormous scales come with a host of challenges that distributed system researchers have focused on over the last four decade. One such key challenge arises from the inherent tension between fault-tolerance, performance, and consistency, elegantly captured by the CAP impossibility theorem [4]. As systems grow in size, the data they hold must be replicated for reasons of both performance (to mitigate the inherent latency of widely distributed systems) and fault-tolerance (to avoid service interruption in the presence of faults). Replicated data is unfortunately difficult to keep consistent: strong consistency, such as linearizability or sequential consistency, is particularly expensive to implement in large-scale systems, and cannot be simultaneously guaranteed together with availability, when using a failure-prone network [4].

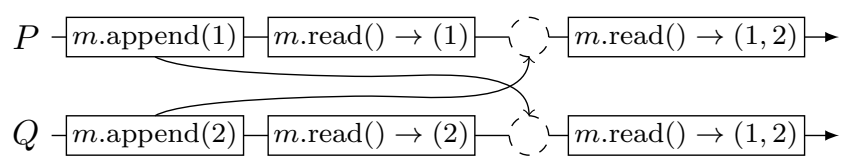

Fig. 1: An eventually consistent append-only queue.

The cost and limitations of strong consistency have prompted an increased interest in weaker consistency conditions for large-scale systems, such as PRAM or causal consistency [5], [6], [7]. Among these conditions, eventual consistency [8], [9] aims to strike a balance between agreement, speed, and dynamicity within a system. Intuitively, eventual consistency allows the replicas of a distributed shared object to temporarily diverge, as long as they eventually converge back to a unique global state.

Formally, this global consistent state should be reached once updates on the object stop (with additional constraints usually linking the object's final value to its sequential specification) [10]. In a practical system, a consistent state should be reached every time updates stop for long enough [3]. How long is long enough depends on the properties of the underlying communication service, notably on its latency and ordering guarantees. These two key properties stand in a natural tradeoff, in which latency can be traded off for better (probabilistic) ordering properties [11], [12], [13]. This inherent tension builds a picture in which an eventually consistent object must find a compromise between speed (how fast are changes visible to other nodes) and consistency (to which extend do different nodes agree on the system's state).

Figure 1, for instance, shows the case of a distributed append-only queue ${ }^{1} m$ manipulated by two processes $P$ and $Q$. $m$ supports two operations: $\operatorname{append}(x)$, which appends an integer $x$ to the queue, and $\operatorname{read}()$, which returns the current content of $m$. In Figure 1, both $P$ and $Q$ eventually converge

\footnotetext{
${ }^{1}$ An append-only queue may for instance be used to implement a distributed blockchain ledger in cryptocurrency systems, or to realize a distributed log.
} 
to the same consistent global state $(1,2)$, that includes both modifications: $m$.append(1) by $P$ and $m$.append(2) by $Q$, in this order. However, $Q$ experiences a temporary inconsistent state when it reads (2): this read does not "see" the append operation by $P$ which has been ordered before $m$.append $(2)$, and is thus inconsistent with the final state $(1,2)^{2} . Q$ could increase the odds of avoiding this particular inconsistency by delaying its first read operation, which would augment its chances of receiving information regarding $P$ 's append operation on time (dashed circle). Such delays improve consistency, but reduce the speed of change propagation across replicas, and must be chosen with care.

Most existing solutions to eventual consistency resolve this tension between speed and consistency by applying one tradeoff point uniformly to all the nodes in a system [3], [11]. However, as systems continue to grow in size and expand in geographic span, they become more diverse, and must cater for diverging requirements. In this paper, we argue that this heterogeneity increasingly call for differentiated consistency levels in large-scale systems. This observation has been made by other researchers, who have proposed a range of hybrid consistency conditions over the years [14], [15], [16], [17], but none of them has so far considered how eventual consistency on its own could offer differentiated levels of speed and consistency within the same system.

Designing such a protocol raises however an important methodological point: how to measure consistency. Consistency conditions are typically formally defined as predicates on execution histories; a system execution is thus either consistent, or it is not. However, practitioners using eventual consistency are often interested in the current level of consistency of a live system, i.e., how far the system currently is from a consistent situation. Quantitatively measuring a system's inconsistencies is unfortunately not straightforward: some practical works [3] measure the level of agreement between nodes, i.e., how many nodes see the same state, but this approach has little theoretical grounding and can thus lead to paradoxes. For instance, returning to Figure 1, if we assume a large number of nodes (e.g., $Q_{1}, \ldots, Q_{n}$ ) reading the same inconsistent state $(2)$ as $Q$, the system will appear close to agreement (many nodes see the same state), although it is in fact largely inconsistent.

To address the above challenges, this paper makes the following contributions:

- We propose a novel consistency mechanism, termed UPS (for Update-Query Consistency with Primaries and Secondaries), that provides different levels of eventual consistency within the same system (Sections III-B,III-C). UPS combines the update-query consistency protocol proposed in [10] with a two-phase epidemic broadcast protocol (called GPS) involving two types of nodes: Primary and Secondary. Primary nodes (the elite) seek to receive object modifications as fast as possible while $\mathrm{Sec}$ -

\footnotetext{
${ }^{2}$ This inconsistency causes in particular $Q$ to observe an illegal state transition from $(2)$ to $(1,2)$.
}

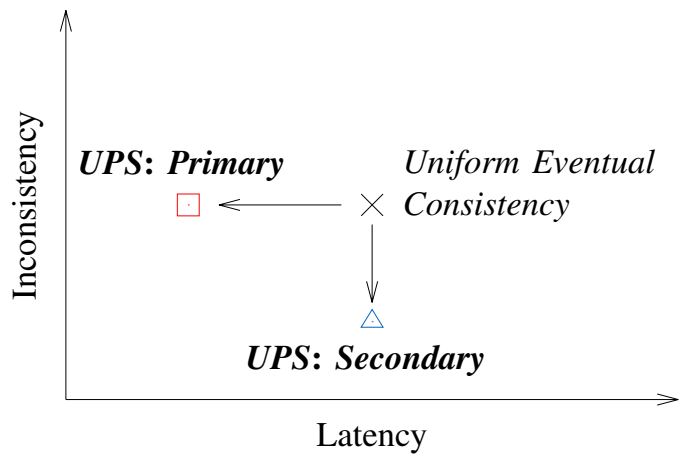

Fig. 2: Aimed consistency/latency trade-off in UPS.

ondary nodes (the masses) strive to minimize the amount of temporary inconsistencies they perceive (Figure 2).

- We formally analyze the latency behavior of the GPSpart of UPS by providing closed-form approximations for the latency incurred by Primary and Secondary nodes (Section III-D).

- We introduce a novel consistency metric that enables us to quantify the amount of inconsistency experienced by Primary and Secondary nodes executing UPS (Section IV).

- We experimentally evaluate the performance of UPS by measuring its consistency and latency properties in largescale simulated networks of one million nodes (Section V). We show in particular that the cost paid by each class of nodes is in fact very small compared to an undifferentiated system: Primary nodes experience a lower latency with levels of inconsistency that are close to those of undifferentiated nodes, while Secondary nodes observe less inconsistencies at a minimal latency cost.

\section{BACKGROUND AND PROBLEM STATEMENT}

\section{A. Update Consistency}

As we hinted at, in Section I, eventual consistency requires replicated objects to converge to a globally consistent state when update operations stop for long enough. By itself, this condition turns out to be too weak as the convergence state does not need to depend on the operations carried out on the object. For this reason, actual implementations of eventually consistent objects refine eventual consistency by linking the convergence state to its sequential specification.

In this paper, we focus on one such refinement, Update consistency [10]. Let us consider the append-only queue object of Figure 1. Its sequential specification consists of two operations:

- append $(x)$, appends the value $x$ at the end of the queue;

- $\operatorname{read}()$, returns the sequence of all the elements ever appended, in their append order.

When multiple distributed agents update the queue, update consistency requires the final convergence state to be the result of a total ordering of all the append operations which respects the program order. For example, the scenario in Figure 1 
satisfies update consistency because the final convergence state results from the ordering $<m$.append(1), $m$.append $(2)>$, which itself respects the program order. An equivalent definition [10] states that an execution history respects update consistency if it contains an infinite number of updates or if it is possible to remove a finite number of reads from it, so that the resulting pruned history is sequentially consistent. In Figure 1, we achieve this by removing the read operation that returns 2 .

Algorithm 1 shows an algorithm from [10] that implements the update-consistent append-only queue of Figure 1. Unlike CRDTs [9] that rely on commutative ("conflict-free") operations, Algorithm 1 exploits a broadcast operation together with Lamport Clocks, a form of logical time-stamps that makes it possible to reconstruct a total order of operations after the fact [10]. Relying on this after-the-fact total order allows update consistency to support non-commutative operations, like the queue in this case.

\section{B. Problem Statement}

The key feature of update consistency lies in the ability to define precisely the nature of the convergence state reached once all updates have been issued. However, the nature of temporary states also has an important impact in practical systems. This raises two important challenges. First, existing systems address the consistency of temporary states by implementing uniform constraints that all the nodes in a system must follow [18]. But different actors in a distributed application may have different requirements regarding the consistency of these temporary states. Second, even measuring the level of inconsistency of these states remains an open question. Existing systems-oriented metrics do not take into account the ordering of update operations (append in our case) [3], [19], [20], [21], while theoretical ones require global knowledge of the system [22] which makes them impractical at large scale.

In the following sections, we address both of these challenges. First we propose a novel broadcast mechanism that, together with Algorithm 1, satisfies update consistency, while supporting differentiated levels of consistency for read operations that occur before the convergence state. Specifically, we exploit the evident trade-off between speed of delivery and consistency, and we target heterogeneous populations consisting of an elite of Primary nodes that should receive fast, albeit possibly inconsistent, information, and a mass of Secondary nodes that should only receive stable consistent information, albeit more slowly. Second, we propose a novel metric to measure the level of inconsistency of an append-only queue, and use it to evaluate our protocol.

\section{The GPS BROADCAST PROTOCOL}

\section{A. System Model}

We consider a large set of nodes $p_{1}, \ldots, p_{N}$ that communicate using point-to-point messages. Any node can communicate with any other node, given its identifier. We use probabilistic algorithms in the following that are naturally robust to crashes and message losses, but do not consider
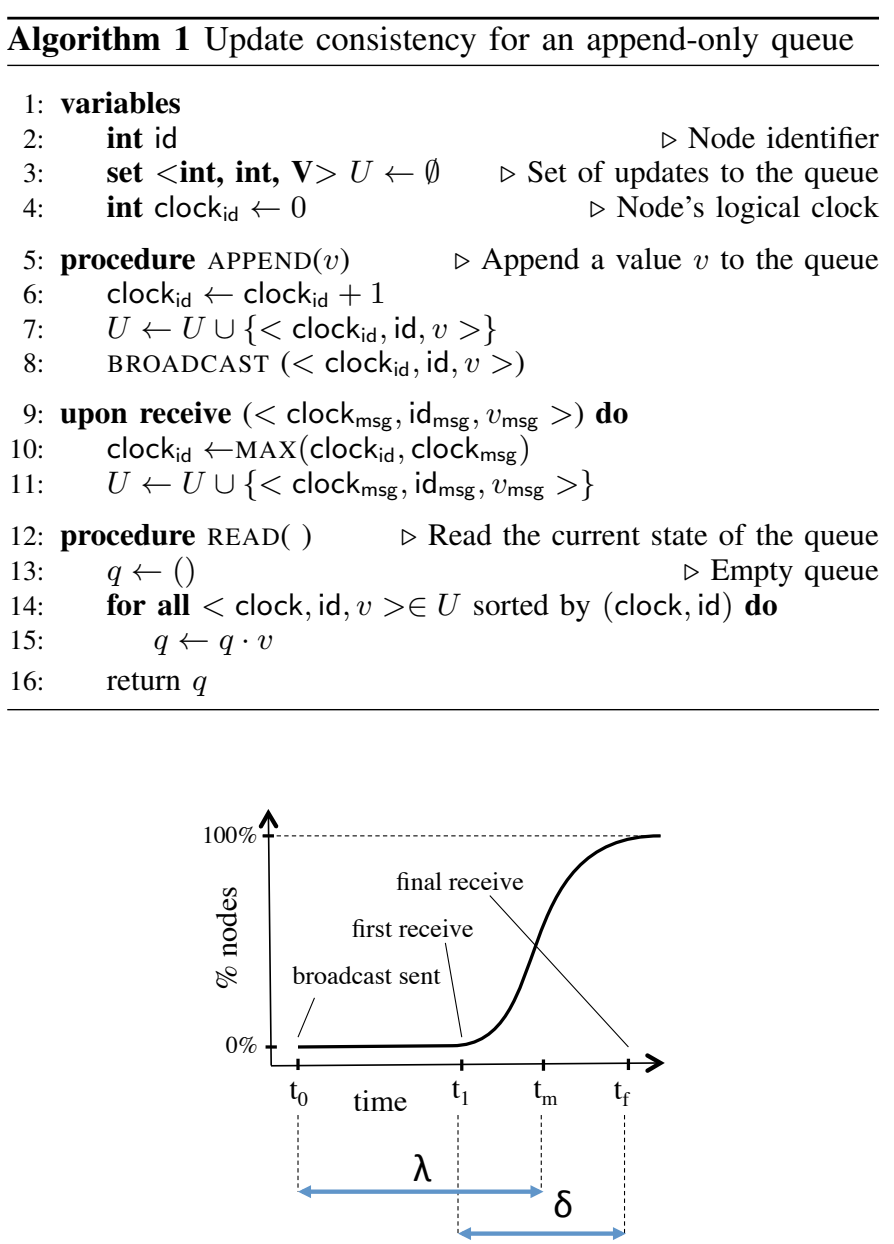

Fig. 3: Two sorts of speeds: latency $(\lambda)$ and jitter $(\delta)$.

these aspects in the rest of the paper for simplicity. Nodes are categorized in two classes: a small number of Primary nodes (the elite) and a large number of Secondary nodes (the masses). The class of a node is an application-dependent parameter that captures the node's requirements in terms of update consistency: Primary nodes should perceive object modification as fast as possible, while Secondary nodes should experience as few inconsistencies as possible.

\section{B. Intuition and Overview}

We have repeatedly referred to the inherent trade-off between speed and consistency in eventually consistent systems. On deeper examination, this trade-off might appear counterintuitive: if Primary nodes receive updates faster, why should not they also experience higher levels of consistency? This apparent paradox arises because we have so far silently confused speed and latency. The situation within a largescale broadcast is in fact more subtle and involves two sorts of speeds (Figure 3): latency ( $\lambda$, shown as an average over all nodes in the figure) is the time a message $m$ takes to reach individual nodes, from the point in time of $m$ 's sending $\left(t_{0}\right)$. Jitter $(\delta)$, by contrast, is the delay between the first $\left(t_{1}\right)$ 


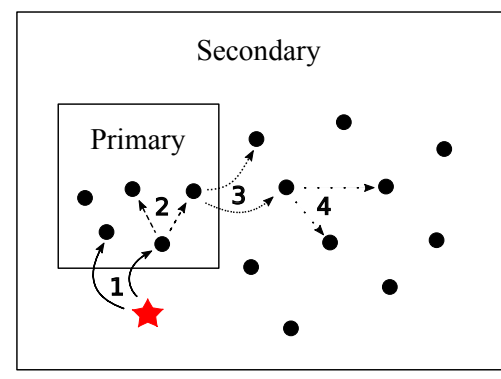

Fig. 4: Model of GPS and path of an update in the system.

and the last receipt $\left(t_{f}\right)$ of a broadcast. (In most large-scale broadcast scenarios, $t_{0}-t_{1}$ is small, and the two notions tend to overlap.) Inconsistencies typically arise in Algorithm 1 when some updates have only partially propagated within a system, and are thus predominantly governed by the jitter $\delta$ rather than the average latency $\lambda$.

The gossip-based broadcast protocol we propose, Gossip Primary-Secondary (GPS), exploits this distinction and proposes different $\delta / \lambda$ trade-offs. Primary nodes have a reduced $\lambda$ (thus increasing the speed at which updates are visible) but also a slightly increased $\delta$, while Secondary nodes have a reduced $\delta$ (thus increasing consistency by lowering the probability for a node to receive updates in the wrong order) but at the cost of a slightly higher $\lambda$.

More precisely, GPS uses the set of Primary nodes as a sort of message "concentrator" that accumulates copies of an update $u$ before collectively forwarding it to Secondary nodes. The main phases of this sequence is shown in Figure 4:

1) A new update $u$ is first sent to Primary nodes (1);

2) Primary nodes disseminate $u$ among themselves (2);

3) Once most Primary nodes have received $u$, they forward it to Secondary nodes (3);

4) Finally, Secondary nodes disseminate $u$ among themselves (4).

A key difficulty in this sequence consists in deciding when to switch from Phase 2 to $\mathbf{3}$. A collective, coordinated transition would require some global synchronization mechanism, a costly and generally impracticable solution in a very large system. Instead, GPS relies on a less accurate but more scalable local procedure based on broadcast counts, which enables each Primary node to decide locally when to start forwarding to secondaries.

\section{The GPS Algorithm}

The pseudo-code of GPS is shown in Algorithm 2. GPS follows the standard models of reactive epidemic broadcast protocols [23], [24]. Each node keeps a history of the messages received so far (in the $R$ variable, line 8 ), and decide whether to re-transmit a received broadcast to fanout other nodes based on its history. Contrary to a standard epidemic broadcast, however, GPS handles Primary and Secondary nodes differently.

- First, GPS uses two distinct Random Peer Sampling protocols (RPS) [25] (lines 10-11) to track the two classes
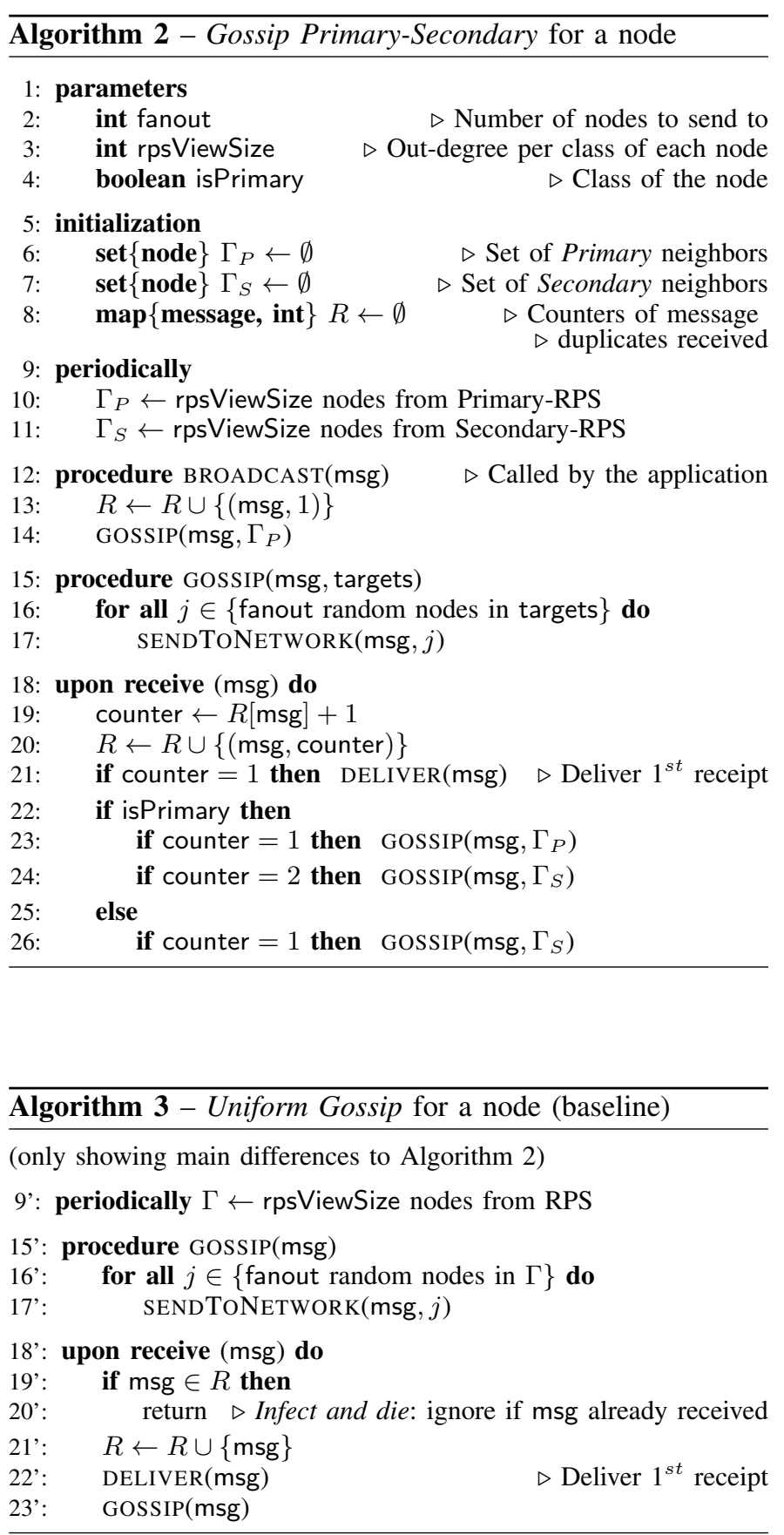

of nodes. Both Primary and Secondary nodes use the RPS view of their category to re-transmit a message they receive for the first time to fanout other nodes in their own category (lines 23 and 24), thus implementing Phases 2 and 4.

- Second, GPS handles retransmissions differently depending on a node's class (Primary or Secondary). Primary nodes use the inherent presence of message duplicates in gossip protocols, to decide locally when to switch from Phase 2 to 3. More specifically, each node keeps 
count of the received copies of individual messages (lines 8, 13, 20). Primary nodes use this count to detect duplicates, and forward a message to fanout Secondary nodes (line 24) when a duplicate is received for the first time, thus triggering Phase 3.

We can summarize the behavior of both classes by saying that Primary nodes infect twice and die, whereas Secondary nodes infect and die. For comparison, a standard infect and die gossip without classes (called Uniform Gossip in the following), is shown in Algorithm 3. We will use Uniform Gossip as our baseline for our analysis (Section III-D) and our experimental evaluation (Section V).

\section{Analysis of GPS}

In the following we compare analytically the expected performance of GPS and compare it to Uniform Gossip in terms of message complexity and latency.

Our analysis uses the following parameters:

- Network $N$ of size: $|N| \in \mathbb{N}$;

- Fanout: $f \in \mathbb{N}$;

- Density of Primary nodes: $d \in \mathbb{R}_{[0,1]}$ (assumed $\leq 1 / f$ ).

Uniform Gossip uses a simple infect and die procedure. For each unique message it receives, a node will send it to $f$ other nodes. If we consider only one source, and assume that most nodes are reached by the message, the number of messages exchanged in the system can be estimated as

$$
|m s g|_{\text {uniform }} \approx f \times|N| .
$$

In the rest of our analysis, we assume, following [26], that the number of rounds needed by Uniform Gossip to infect a high proportion of nodes can be approximated by the following expression when $N \rightarrow \infty$ :

$$
\lambda_{\text {uniform }} \approx \log _{f}(|N|)+C,
$$

where $C$ is a constant, independent of $\mathrm{N}$.

GPS distinguishes two categories of nodes: Primary nodes and Secondary nodes, noted $P$ and $S$, that partition $N$. The density of Primary nodes, noted $d$, defines the size of both subsets:

$$
|P|=d \times|N|, \quad|S|=(1-d) \times|N| .
$$

Primary nodes disseminate twice, while Secondary nodes disseminate once. Expressed differently, each node disseminates once, and Primary nodes disseminate once more. Applying the same estimation as for Uniform Gossip gives us:

$$
\begin{aligned}
|m s g|_{G P S} & \approx f \times|N|+f \times|P| \\
& \approx f \times|N|+f \times d \times|N| \\
& \approx(1+d) \times f \times|N| \\
& \approx(1+d) \times|m s g|_{\text {uniform }} .
\end{aligned}
$$

(1) and (3) show that GPS only generates $d$ times more messages than Uniform Gossip, with $d \in \mathbb{R}_{[0,1]}$. For instance, having $1 \%$ Primary nodes in the network means having only $1 \%$ more messages compared to Uniform Gossip.
If we now turn to the latency behavior of GPS, the latency of the Primary nodes is equivalent to that of Uniform Gossip executing on a sub-network composed only of $d \times|N|$ nodes, i.e.,

$$
\lambda_{P} \approx \log _{f}(d \times|N|)+C .
$$

Combining (2) and (4), we conclude that Primary nodes gain $\log _{f}(d)$ rounds compared to nodes in Uniform Gossip:

$$
\Delta \lambda_{P} \approx-\log _{f}(d) \text {. }
$$

Considering now Secondary nodes, their latency can be estimated as a sum of three elements:

- the latency of Primary nodes;

- an extra round for Primary nodes to receive messages a second time;

- the latency of a Uniform Gossip among Secondary nodes with $d \times|N|$ nodes, corresponding to the Primary nodes, already infected;

which we approximate for $d \ll 1$ as

$$
\begin{aligned}
\lambda_{S} & \approx \log _{f}(d \times|N|)+1+\log _{f}\left(\frac{(1-d) \times|N|}{d \times|N|}\right)+2 C, \\
& \approx \log _{f}((1-d) \times|N|)+1+2 C .
\end{aligned}
$$

In summary, this analysis shows that GPS only generates a small number of additional messages, proportional to the density $d$ of Primary nodes, and that the latency cost paid by Secondary is bounded by a constant value $(1+C)$ that is independent of the Primary density $d$.

\section{COnsistency Metric}

Our second contribution is a novel metric that measures the consistency level of the temporary states of an updateconsistent execution. As discussed in Section II, existing consistency metrics fall short either because they do not capture the ordering of operations, or because they cannot be computed without global system knowledge. Our novel metric satisfies both of these requirements.

\section{A. A General Consistency Metric}

We start by observing that the algorithm for an updateconsistent append-only queue (Algorithm 1) guarantees that all its execution histories respect update consistency. To measure the consistency level of temporary states, we therefore evaluate how the history deviates from a stronger consistency model, sequential consistency [27]. An execution respects sequential consistency if it is equivalent to some sequential (i.e., totally ordered) execution that contains the same operations, and respects the sequential (process) order of each node.

Since update consistency relies itself on a total order, the gist of our metric consists in counting the number of read operations that do not conform with a total order of updates that leads to the final convergence state. Given one such total order, we may transform the execution into one that conforms with it by removing some read operations. In general, a data object may reach a given final convergence state by means of different possible total orders, and for each such total order 
we may have different sets of read operations whose removal makes the execution sequentially consistent. We thus count the level of inconsistency by taking the minimum over these two degrees of freedom: choice of the total order, and choice of the set.

More formally, we define the temporary inconsistencies of an execution Ex as a finite set of read operations that, when removed from $E x$, makes it sequentially consistent. We denote the set of all the temporary inconsistencies of execution $E x$ over all compatible total orders by $T I(E x)$. We then define the relative inconsistency $R I$ of an execution $E x$ as the minimal number of read operations that must be removed from $E x$ to make it sequentially consistent.

$$
R I(E x)=\left\{\begin{array}{cl}
\min _{E \in T I(E x)}|E| & \text { if } T I(E x) \neq \emptyset \\
+\infty & \text { otherwise }
\end{array}\right.
$$

For example, in Figure 1, if we consider the total order $<m$.append(1), $m$.append(2), m.read(1), m.read(2), $m \cdot \operatorname{read}(1,2), m \cdot \operatorname{read}(1,2)>$ then we need to remove both $m$. read(1) and $m$.read(2) to make the execution sequentially consistent. Removing only $m$.read(2), instead, suffices to make the execution sequentially consistent with respect to $<m$.append(1), $m \cdot \operatorname{read}(1), m$.append(2),$m \cdot \operatorname{read}(2)$, $m \cdot \operatorname{read}(1,2), m \cdot \operatorname{read}(1,2)>$. Therefore the level of inconsistency of the execution in Figure 1 is 1.

The metric $R I$ is particularly adapted to compare the consistency level of implementations of update consistency: the lower, the more consistent. In the best case scenario where $E x$ is sequentially consistent, $T I(E x)$ is a singleton containing the empty set, resulting in $R I(E x)=0$. In the worst case scenario where the execution never converges (i.e., some nodes indefinitely read incompatible local states), every set of reads that needs to be removed to obtain a sequentially consistent execution is infinite. Since TI only contains finite sets of reads, $T I(E x)=\emptyset$ and $R I(E x)=+\infty$.

\section{B. The Case of our Update-Consistent Append-Only Queue}

In general, $R I(E x)$ is complex to compute: it is necessary to consider all possible total orders of events that can fit for sequential consistency and all possible finite sets of reads to check whether there are temporary inconsistencies. But in the case of an append-only queue implemented with Algorithm 1, we can easily show that there exists exactly one minimal set of temporary inconsistencies.

To understand why, we first observe that the append operation is non-commutative. This implies that there exists a single total order of append operations that yields a given final convergence state. Second, Algorithm 1 guarantees that the size of the successive sequences read by a node can only increase and that read operations always reflect the writes made on the same node. Consequently, in order to have a sequentially consistent execution, it is necessary and sufficient to remove all the read operations that return a sequence that is not a prefix of the sequence read after convergence. These read operations constitute the minimal set of temporary inconsistencies $T I_{\min }$.

\section{EXPERIMENTAL RESUlTS}

We perform the evaluation of UPS via PeerSim [28], a well-known Peer-to-Peer simulator. A repository containing the code and the results is available on-line ${ }^{3}$. To assess the trade-offs between consistency level and latency as well as the overhead of UPS, we focus the evaluation on three metrics:

- the level of consistency of the replicated object;

- the latency of messages;

- the overhead in number of messages.

\section{A. Methodology}

1) Network Settings: We use a network of 1 million nodes, a fanout of 10 and an RPS view size of 100 . These parameters yield a broadcast reliability (i.e., the probability that a node receives a message) that is above $99.9 \%$. Reliability could be further increased with a higher fanout [23], but these parameters, because they are all powers of 10 , make it convenient to understand our experimental results.

We use density values of Primary nodes of: $10^{-1}, 10^{-2}$ and $10^{-3}$. According to Equation 5 in Section III-D, we expect to see a latency gain for Primary nodes of 1,2 , and 3 rounds respectively. We evaluate four protocol configurations: one for Uniform Gossip (baseline), and three for UPS with the three above densities, then run each configuration 25 times and record the resulting distribution.

2) Scenario: We consider a scenario where all nodes share an instance of an update-consistent append-only queue, as defined in Section II-A. Following the definition of update consistency, nodes converge into a strongly consistent state once they stop modifying the queue.

We opt for a scenario where 10 append $(x)$ operations, with $x \in \mathbb{Z}$, are performed on the queue by 10 random nodes, over the first 10 rounds of the simulations at the frequency of one update per round. In addition, all nodes repeatedly read their local copy of the queue in each round.

We expect the system to experience two periods: first, a temporary situation during which updates are issued and disseminated (simulating a system continuously performing updates), followed by a stabilized state after updates have finished propagating and most nodes have converged to a strongly consistent state.

3) Consistency Metric: Our experimental setting enables us to further refine the generic consistency metric we introduced in Section IV. Since nodes perform a finite number of update operations and each node reads the state of the queue at each round, we define a per-round metric to compare the evolution of the inconsistency of Primary and Secondary nodes through time. For each round $r$, we define the sets $R_{P}(r)$ and $R_{S}(r)$ as the sets of all the read operations performed at round $r$ by the Primary and Secondary nodes respectively. Then we define per-round inconsistency, $\operatorname{Incons}_{P}(r)$ and $\operatorname{Incons}_{S}(r)$, as the proportion of Primary and Secondary nodes that see an inconsistent read at round $r$ (i.e., the corresponding reads are in $\left.T I_{\min }\right)$.

\footnotetext{
${ }^{3}$ https://gforge.inria.fr/projects/pgossip-exp/
} 


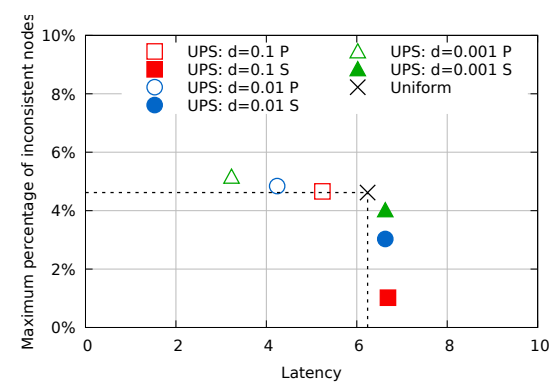

(a) Experimental trade-offs between consistency and latency (closer to the bottom left corner is better). A big gain on one side of the balance incurs a small penalty on the other side of the balance for a class.

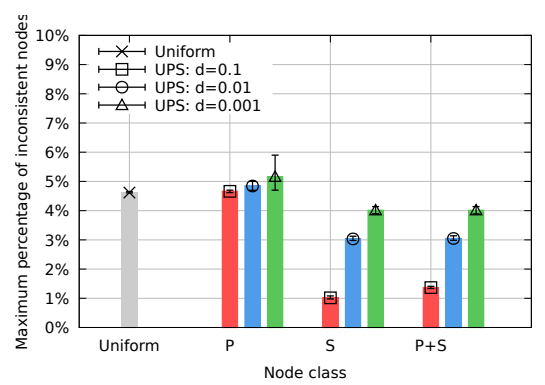

(b) Consistency trade-off in UPS (lower is better). Secondary nodes are much more consistent than nodes in the baseline while Primary nodes only experience a small consistency penalty. The consistency of both Primary and Secondary nodes improves as the density of Primary nodes increases.

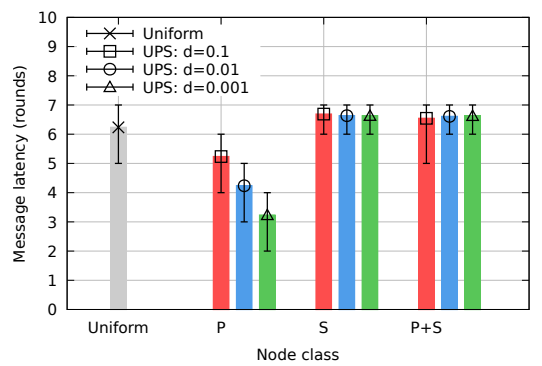

(c) Latency trade-off in UPS (lower is better). Primary nodes latency is lower than that of the baseline; the less Primary nodes, the lower their latency. Secondary nodes remain half a round slower than the baseline, regardless of the density of Primary nodes in the network.

Fig. 5: Consistency and latency trade-off in UPS. Primary nodes are faster and a bit less consistent, while Secondary are more consistent and a bit slower. The top of the bars is the mean while the ends of the error bars are the 5th and 95th percentiles.

$$
\begin{gathered}
\operatorname{Incons}_{P}(r)=\frac{\left|T I_{\text {min }} \cap R_{P}(r)\right|}{d \times|N|} \\
\text { Incons }_{S}(r)=\frac{\left|T I_{\text {min }} \cap R_{S}(r)\right|}{(1-d) \times|N|} \\
\text { Incons }_{P+S}(r)=d \times \text { Incons }_{P}(r)+(1-d) \times \text { Incons }_{S}(r)
\end{gathered}
$$

In the following, we focus on these instantaneous per-round metrics, which provide an equivalent but more accurate view than the simple relative inconsistency of an experimental run, $R I(E x)$. We can in fact compute $R I(E x)$ as follows.

$$
R I(E x)=|N| \times \sum_{r=0}^{\infty}\left(\text { Incons }_{P+S}(r)\right) .
$$

4) Plots: Unless stated otherwise, plots use boxes and whiskers to represent the distribution of measures obtained for the represented metric. For the inconsistency measures, the distribution is over all runs. For the latency measures, the distribution is over all nodes within all runs. The end of the boxes show the first and third quartiles, the end of the whiskers represent the minimum and maximum values, while the horizontal bar inside the boxes is the mean. In some cases, the low variability of the results makes it difficult to see the boxes and whiskers.

For clarity purposes, curves are slightly shifted to the right to avoid overlap between them. All the points between rounds $r$ and $r+1$ belong to round $r$. In the following plots, Primary nodes are noted $P$, Secondary nodes are noted $S$ and the system as a whole is noted $P+S$. Since only a small fraction of nodes are Primary nodes, the results of $P+S$ are naturally close to those of $S$.

\section{B. Overall Results}

Figure 5a mirrors Figure 2 discussed in Section I and provides an overview of our experimental results in terms of consistency/latency trade-offs for the different sets of nodes involved in our scenario. Each UPS configuration is shown as a pair of points representing Primary and Secondary nodes: Primary nodes are depicted with hollow shapes, while Secondary nodes use solid symbols. Uniform Gossip is represented by a single black cross. The position on the $x$-axis charts shows the average update latency experienced by each set of nodes, and the $y$-axis their perceived level of inconsistency, taken as the maximum Incons $_{X}(r)$ value measured over all runs.

The figure clearly shows that UPS delivers the differentiated consistency/latency trade-offs we set out to achieve in our introduction: Secondary nodes enjoy higher consistency levels than they would in an uniform update-query consistency protocol, while paying only a small cost in terms of latency. The consistency boost strongly depends on the density of Primary nodes in the network, while the cost in latency does not, reflecting our analysis of Section III-D. Primary nodes present the reverse behavior, with the latency gains of Primary nodes evolving in the reverse direction of the consistency gains of Secondary nodes. We discuss both aspects in more details in the rest of this section.

\section{Consistency Level}

Figure $5 \mathrm{~b}$ details the consistency levels provided by UPS by showing the worst consistency that nodes experience over all simulations. We note an evident improvement of the maximum inconsistency level of Secondary nodes over the baseline and a slight decrease for Primary nodes. In addition, this figure clearly shows the impact of the density of Primary nodes over the consistency of Secondary nodes, and to a lesser extent over the consistency of Primary nodes.

Figures $6 \mathrm{a}, 6 \mathrm{~b}$ and $6 \mathrm{c}$ show the evolution over time of the inconsistency measures $\operatorname{Incons}_{P}(r)$, Incons $S_{S}(r)$ and Incons $_{P+S}(r)$ defined in Section $\mathrm{V}$-A3. We can observe an increase of inconsistencies during the temporary phase for all configurations and a return to a consistent state once every node has received every update. 


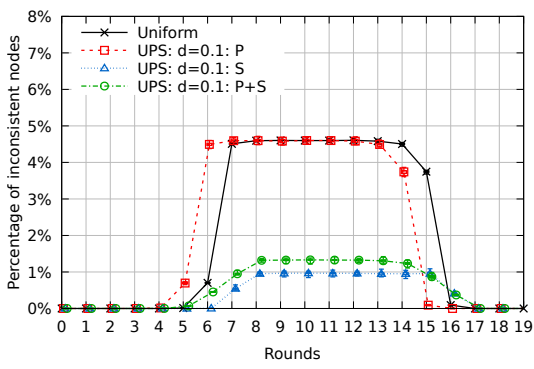

(a) Density $=10^{-1}$

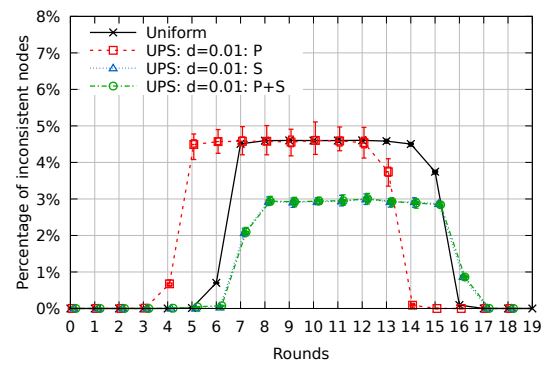

(b) Density $=10^{-2}$

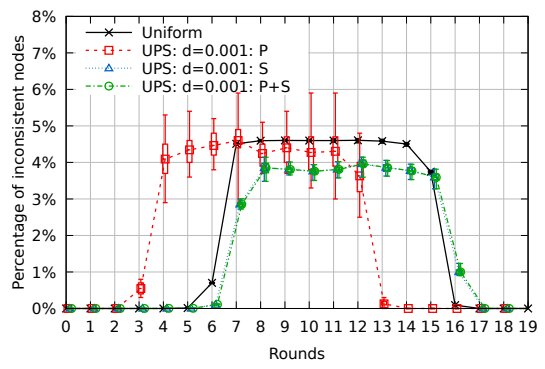

(c) Density $=10^{-3}$

Fig. 6: While updates are being disseminated, Secondary nodes are more consistent than the baseline and Primary nodes are on average as consistent as the baseline. The more Primary nodes are in the system, the more Secondary nodes are consistent. Once updates stop being performed, Primary nodes converge faster to a strongly consistent state.

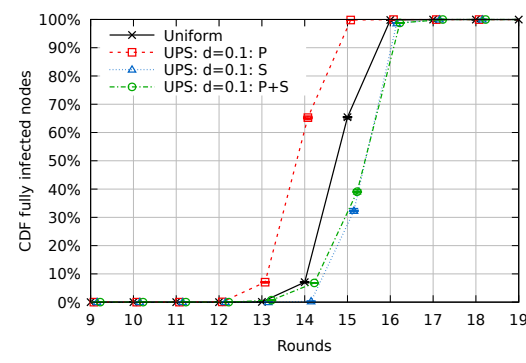

(a) Density $=10^{-1}$

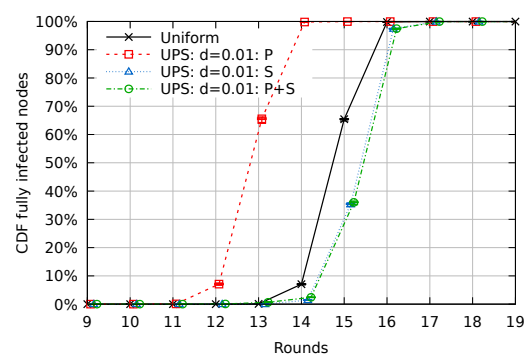

(b) Density $=10^{-2}$

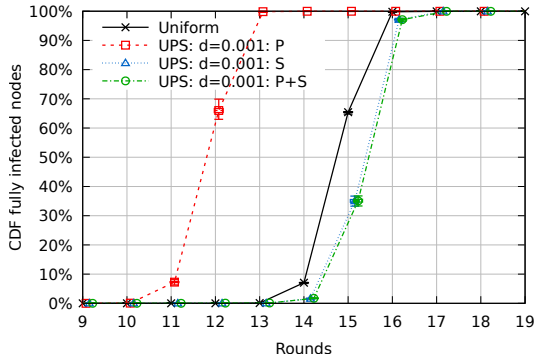

(c) Density $=10^{-3}$

Fig. 7: Primary nodes receive all the updates faster than the baseline: they gain 1,2 and 3 rounds for densities of $10^{-1}, 10^{-2}$ and $10^{-3}$ respectively. Meanwhile Secondary nodes receive all the updates only half a round later on average compared to nodes in the baseline.

During the temporary phase, the inconsistency level of Primary nodes is equivalent to that of nodes in Uniform Gossip. In that phase, around $4.6 \%$ of Primary nodes are inconsistent with a higher variability for lower densities.

Meanwhile, the inconsistency level of Secondary nodes is much lower than that of nodes in Uniform Gossip. The density of Primary nodes plays a key role in this difference; the higher the density, the lower the inconsistency level of Secondary nodes. This level remains under $1.0 \%$ for the highest density but goes up to $4.0 \%$ for the lowest density.

The jitter, as defined in Section III-B, is a good metric to compare the consistency of different sets of nodes: a lower jitter implies a lower probability for a node to receive updates in the wrong order, which in turn leads to a higher consistency. The error bars in Figure 5c provide an approximation of the jitter of a set of nodes since they represent the bounds in latency of $90 \%$ of the nodes in a set. These error bars show that $90 \%$ of Secondary nodes receive updates within 1 round of margin, while the same proportion of Primary nodes and nodes in Uniform Gossip receive updates within 2 rounds of margin. This difference in jitter explains why Secondary nodes are more consistent than Primary nodes and nodes in Uniform Gossip.

Once the dissemination reaches a critical mass of Primary nodes, the infection of Secondary nodes occurs quickly. If the density of Primary nodes is high enough, then it becomes possible for a majority of Secondary nodes to receive the same update at the same time. Since it takes fewer rounds for Secondary nodes to be fully infected compared to Primary nodes, Secondary nodes turn out to be more consistent.

\section{Message Latency}

Figure $5 \mathrm{c}$ represents the distribution of all the values of message latency over all the simulation runs. The three $P+S$ bars and the Uniform bar contain each 250 million message latency values ( 25 runs $\times 1$ million nodes $\times 10$ sources with a reliability above $99.9 \%$ ).

This figure shows that UPS infects Primary nodes faster than Uniform Gossip would. Specifically, Primary nodes obtain a latency gain of 1,2 and 3 rounds with densities of $10^{-1}, 10^{-2}$ and $10^{-3}$ respectively. Secondary nodes, on the other hand, are infected half a round slower than nodes in Uniform Gossip for all density values.

Figures $7 \mathrm{a}, 7 \mathrm{~b}$ and $7 \mathrm{c}$ compare the evolution of the dissemination of updates between Uniform Gossip and UPS with different densities. Again, Primary nodes have a 1, 2 and 3 rounds latency advantage over the baseline while Secondary nodes are no more than a round slower. 
We can also observe this effect on Figure 6 by looking at how fast all the nodes of a class return to a consistent state. We notice similar latency gain for Primary nodes and loss for Secondary nodes.

Overall, the simulation results match the analysis in Section III-D and confirm the latency advantage of Primary nodes over Uniform Gossip (Equation 5) and the small latency penalty of Secondary nodes (Equation 6).

\section{E. Network Overhead}

The number of messages exchanged in the simulated system confirms Equation 3 in Section III-D. Considering the experienced reliability, we observe in $U P S$ an increase in the number of messages of $10^{-1}, 10^{-2}$ and $10^{-3}$ compared to Uniform Gossip for all three densities of $10^{-1}, 10^{-2}$ and $10^{-3}$ respectively.

\section{RELATED WORK}

UPS lies at the crossroad between differentiated consistency and gossip protocols (for the GPS-part). In the following, we review some of the most relevant works from these two areas.

a) Differentiated Consistency: A large number of works have looked at hybrid consistency conditions, originally for distributed shared memory [16], [29], [30], and more recently in the context of geo-distributed systems [14], [15], [17], [31]. Fisheye [15] and RedBlue [17] for instance both propose to implement hybrid conditions for geo-replicated systems. Fisheye consistency provides a generic approach in which nodes that are topologically close satisfy a strong consistency criterion, such as sequential consistency, while remote nodes satisfy a weaker one, such as causal consistency. This formal work focuses exclusively on immediate (i.e., non-eventual) consistency criteria and does not take convergence speed into account. RedBlue consistency offers a trade-off similar to that of UPS, but focuses on operations, rather than nodes, as we do. Blue operations are fast and eventually consistent while red operations are slow and strongly consistent.

b) Measuring Inconsistency: Several papers have proposed metrics to evaluate a system's overall consistency. The approach of Zellag and Kemme [22] detects inconsistencies in cloud services by finding cycles in a dependency graph composed of transactions (nodes) and conflicts between them (edges). Counting cycles in the dependency graph yields a measure of consistency that is formally grounded. It requires however a global knowledge of the system, which makes it difficult to use in practice in large-scale systems. Golab et al. introduced first $\Delta$-atomicity [19], [32] and then $\Gamma$ [20], two metrics that quantify data staleness against Lamportatomicity [33] in key-value store traces. These metrics are not suitable for our problem since they do not take into account the ordering of update operations.

More practical works [3], [21] evaluate consistency by relying on system specific information such as the similarity between different cache levels or the read-after-write latency (the first time a node reads the value that was last written).
Finally, CRDTs [9] remove the need to measure consistency by only supporting operations that cannot create conflicts. This naturally leads to eventual consistency without additional ordering requirements on communication protocols.

c) Biased Gossip Protocols: Many gossip broadcast protocols use biases to accommodate system heterogeneity. However, to the best of our knowledge, GPS is the first such protocol to target heterogeneous consistency requirements.

Directional gossip [34], for instance, favors weakly connected nodes in order to improve its overall reliability. It does not target speed nor consistency, as we do. The work in [35] looks at reducing a broadcast's message complexity by considering two classes of user-defined nodes: good and bad nodes. A new broadcast is disseminated to good nodes first using a reactive epidemic protocol, while bad nodes are reached through a slower periodic push procedure. As a result, the overall number of messages is reduced, at the cost of higher delivery latency for bad nodes. Similarly, Gravitational gossip [36] proposes a multicast protocol with differential reliability to better balance the communication workload between nodes, according to their capacities. Gravitational gossip associates each node with a susceptibility $S_{r}$ and an infectivity $I_{r}$ value that depend on a user-defined quality rating $r$. Nodes of rating $r$ receive a fraction $r$ of the messages before they time out. Gravitational gossip thus offers a cost/reliability trade-off, while GPS considers a consistency/latency tradeoff. Hierarchical gossip [37] also aims to reduce overheads but focuses on those associated with the physical network topology. To this end, it favors gossip targets that are close in the network hierarchy, which leads to a slight decrease in reliability and an increase of delivery latency.

In the context of video streaming, HEAP [38] adapts the fanout of nodes to reduce delivery latency in the presence of heterogeneous bandwidth capabilities. In addition, nodes do not wait for late messages, they simply ignore them. This dropping policy is well adapted to video streaming but cannot be applied to GPS. Finally, epidemic total order algorithms such as EpTO [11] and ecBroadcast [12] can be used to implement (probabilistic) strong consistency conditions, but at the cost of higher latency, and a higher number of messages for EpTO.

\section{CONCLUSION}

We have presented Update-Query Consistency with Primaries and Secondaries (UPS), a novel eventual consistency mechanism that offers heterogeneous properties in terms of data consistency and delivery latency. Primary nodes can deliver updates faster at the cost of a small consistency penalty, while Secondary nodes experience stronger consistency but with a slightly higher latency. Both sets of nodes observe a consistent state with high probability once dissemination completes.

To measure the different levels of consistency observed by Primary and Secondary nodes, we have proposed a novel and scalable consistency metric grounded in theory. This metric 
detects when a node performs read operations that conflict with the sequential specification of its associated object.

We formally analyzed the latency incurred by nodes as well as the overhead in message complexity in GPS, the underlying two-phase epidemic broadcast protocol. We then evaluated both the consistency and latency properties of UPS by simulating a 1 million node network. Results show how the density (fraction) of Primary nodes influences the tradeoff between consistency and latency: lower densities favor fast dissemination to Primary nodes, while higher densities favor higher consistency for Secondary nodes.

Our future plans include deploying UPS in a real system and performing experiments to confront the algorithm to reallife conditions. We also plan to investigate how UPS could be combined with a complementary anti-entropy protocol [39] to reach the last few susceptible nodes and further improve its reliability.

\section{ACKNOWLEDGMENTS}

This work has been partially funded by the Region of Brittany, France, by the Doctoral school of the University of Brittany Loire (UBL) and by the French National Research Agency (ANR) project SocioPlug under contract ANR-13INFR-0003 (http://socioplug.univ-nantes.fr).

\section{REFERENCES}

[1] A. Verma, L. Pedrosa, M. Korupolu, D. Oppenheimer, E. Tune, and J. Wilkes, "Large-scale cluster management at google with borg," in EuroSys. ACM, 2015.

[2] "Google data centers, data center locations," https://www.google.com/ about/datacenters/inside/locations/index.html, accessed Sep. 102015.

[3] H. Lu, K. Veeraraghavan, P. Ajoux, J. Hunt, Y. J. Song, W. Tobagus, S. Kumar, and W. Lloyd, "Existential Consistency: Measuring and Understanding Consistency at Facebook," in SOSP. ACM, 2015.

[4] S. Gilbert and N. Lynch, "Brewer's conjecture and the feasibility of consistent, available, partition-tolerant web services," ACM SIGACT News, vol. 33, no. 2, 2002.

[5] P. Bailis, A. Ghodsi, J. M. Hellerstein, and I. Stoica, "Bolt-on causal consistency," in ACM SIGMOD Int. Conf. on Man. of Data, 2013.

[6] S. Almeida, J. Leitão, and L. Rodrigues, "Chainreaction: a causal+ consistent datastore based on chain replication," in EuroSys. ACM, 2013.

[7] W. Lloyd, M. J. Freedman, M. Kaminsky, and D. G. Andersen, "Don't settle for eventual: scalable causal consistency for wide-area storage with COPS," in SOSP. ACM, 2011.

[8] W. Vogels, "Eventually Consistent," CACM, vol. 52, no. 1, Jan. 2009.

[9] M. Shapiro, N. Preguiça, C. Baquero, and M. Zawirski, "Conflict-Free Replicated Data Types," in SSS, 2011.

[10] M. Perrin, A. Mostefaoui, and C. Jard, "Update Consistency for Waitfree Concurrent Objects," in IPDPS. IEEE, 2015.

[11] M. Matos, H. Mercier, P. Felber, R. Oliveira, and J. Pereira, "EpTO: An Epidemic Total Order Algorithm for Large-Scale Distributed Systems," in Middleware. ACM, 2015.

[12] R. Baldoni, R. Guerraoui, R. R. Levy, V. Quéma, and S. T. Piergiovanni, "Unconscious Eventual Consistency with Gossips," in SSS, 2006.

[13] A. Sousa, J. Pereira, F. Moura, and R. Oliveira, "Optimistic total order in wide area networks," in SRDS. IEEE, 2002.

[14] C. Xie, C. Su, M. Kapritsos, Y. Wang, N. Yaghmazadeh, L. Alvisi, and P. Mahajan, "Salt: Combining acid and base in a distributed database," in OSDI. USENIX, 2014.

[15] R. Friedman, M. Raynal, and F. Taïani, "Fisheye Consistency: Keeping Data in Synch in a Georeplicated World," in NETYS, 2015.

[16] R. Friedman, "Implementing hybrid consistency with high-level synchronization operations," Dist. Comp., vol. 9, no. 3, 1995.
[17] C. Li, D. Porto, A. Clement, J. Gehrke, N. Preguiça, and R. Rodrigues, "Making geo-replicated systems fast as possible, consistent when necessary," in OSDI. USENIX, 2012.

[18] S. Burckhardt, A. Gotsman, H. Yang, and M. Zawirski, "Replicated data types: Specification, verification, optimality," SIGPLAN Not., vol. 49, no. $1,2014$.

[19] W. Golab, X. Li, and M. A. Shah, "Analyzing consistency properties for fun and profit," in PODC. ACM, 2011.

[20] W. Golab, M. R. Rahman, A. AuYoung, K. Keeton, and I. Gupta, "Client-centric benchmarking of eventual consistency for cloud storage systems," in ICDCS. IEEE, 2014.

[21] S. Patil, M. Polte, K. Ren, W. Tantisiriroj, L. Xiao, J. López, G. Gibson, A. Fuchs, and B. Rinaldi, "YCSB++: benchmarking and performance debugging advanced features in scalable table stores," in Symp. on Cloud Comp. (SoCC). ACM, 2011.

[22] K. Zellag and B. Kemme, "How consistent is your cloud application?" in Symp. on Cloud Comp. (SoCC). ACM, 2012.

[23] A.-M. Kermarrec, L. Massoulie, and A. Ganesh, "Probabilistic reliable dissemination in large-scale systems," IEEE TPDS, vol. 14, no. 3, 2003.

[24] F. Taïani, S. Lin, and G. S. Blair, "GossipKit: A unified componentframework for gossip," IEEE TSE, vol. 40, no. 2.

[25] M. Jelasity, S. Voulgaris, R. Guerraoui, A.-M. Kermarrec, and M. Van Steen, "Gossip-based peer sampling," ACM ToCS, vol. 25, no. 3, 2007.

[26] P. T. Eugster, R. Guerraoui, A.-M. Kermarrec, and L. Massoulié, "From epidemics to distributed computing," IEEE computer, vol. 37, no. LPDARTICLE-2006-004, pp. 60-67, 2004.

[27] L. Lamport, "How to make a multiprocessor computer that correctly executes multiprocess programs," IEEE ToC, vol. 100, no. 9, 1979.

[28] A. Montresor and M. Jelasity, "PeerSim: A scalable P2P simulator," in P2P. IEEE, 2009.

[29] H. Attiya and R. Friedman, "Limitations of fast consistency conditions for distributed shared memories," Inf. Proc. Letters, vol. 57, no. 5, 1996.

[30] P. Keleher, A. L. Cox, and W. Zwaenepoel, "Lazy release consistency for software distributed shared memory," in ISCA. ACM, 1992.

[31] D. B. Terry, V. Prabhakaran, R. Kotla, M. Balakrishnan, M. K. Aguilera, and H. Abu-Libdeh, "Consistency-based service level agreements for cloud storage," in SOSP. ACM, 2013.

[32] M. R. Rahman, W. Golab, A. AuYoung, K. Keeton, and J. J. Wylie, "Toward a principled framework for benchmarking consistency," in HotDep. USENIX, 2012.

[33] L. Lamport, "On interprocess communication," Distributed Computing, vol. 1, no. 2, 1986

[34] M.-J. Lin and K. Marzullo, "Directional Gossip: Gossip in a Wide Area Network," in EDCC, 1999.

[35] N. Carvalho, J. Pereira, R. Oliveira, and L. Rodrigues, "Emergent Structure in Unstructured Epidemic Multicast," in DSN, 2007.

[36] K. Hopkinson, K. Jenkins, K. Birman, J. Thorp, G. Toussaint, and M. Parashar, "Adaptive Gravitational Gossip: A Gossip-Based Communication Protocol with User-Selectable Rates," IEEE TPDS, vol. 20 no. $12,2009$.

[37] I. Gupta, A.-M. Kermarrec, and A. Ganesh, "Efficient and adaptive epidemic-style protocols for reliable and scalable multicast," IEEE TPDS, vol. 17, no. 7, 2006

[38] D. Frey, R. Guerraoui, A.-M. Kermarrec, B. Koldehofe, M. Mogensen, M. Monod, and V. Quéma, "Heterogeneous Gossip," in Middleware. ACM, 2009.

[39] A. Demers, D. Greene, C. Hauser, W. Irish, J. Larson, S. Shenker, H. Sturgis, D. Swinehart, and D. Terry, "Epidemic Algorithms for Replicated Database Maintenance," in PODC. ACM, 1987. 